\title{
Malformación arteriovenosa en cavidad oral: A propósito de un caso y revisión de la literatura
}

\author{
Arteriovenous malformation in the oral cavity: \\ A case report and a literature review
}

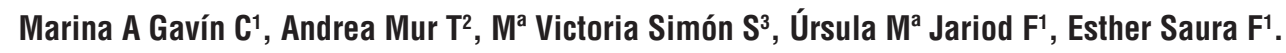

\begin{abstract}
RESUMEN
Las malformaciones arteriovenosas (malformaciones A-V) de alto flujo en la cavidad oral no son una patología frecuente, existen pocos datos publicados sobre su tratamiento y sigue siendo muy controvertido debido a la alta tasa de complicaciones vitales que conIleva y la alta tasa de recurrencia. El único tratamiento curativo es la resección radical, con las consecuencias no sólo vitales, sino también desfigurativas que supone. Presentamos un caso de malformación A-V congénita en región submandibular, suelo de boca derecho y lengua, que tras varios episodios de dolor y aumento de tamaño, sin realizar ninguna medida agresiva, en el último control se observa trombosis espontánea de la misma. En este caso, la evolución con un tratamiento expectante sin cirugía, ha sido la trombosis con mejoría clínica, sin presentar nuevo episodio de aumento de tamaño, ni sangrado doce meses después, a pesar de seguir con una lesión de gran tamaño en el suelo de la boca.

Palabras clave: Malformación arteriovenosa, cavidad oral, lesión congénita tratamiento conservador.
\end{abstract}

\begin{abstract}
The high-flow arteriovenous malformations (A-V malformations) in oral cavity are not a common disease, there are few published data on treatment and it remains highly controversial because of the high rate of vital complications and the high rate of recurrence. The only curative treatment is radical resection, with not only vital consequences but also the disfigurement involved. We present a case of congenital $A-V$ malformation in right submandibular region, floor of the mouth and tongue. After several episodes of pain and enlargement, in the last control without any aggressive action, the lesion presented spontaneous thrombosis. In this case, despite following with a large lesion on the floor mouth, the evolution with an expectant non-surgical treatment has been the thrombosis with clinical improvement, without presenting new episode of enlargement, nor bleeding twelve months later.
\end{abstract}

Key words: Arteriovenous malformation, oral cavity, treatment follow.

\footnotetext{
1 Médico Departamento de Cirugía Oral y Maxilofacial. Hospital Universitario Miguel Servet, Zaragoza.

2 Facultativo Especialista de Área en el Departamento de Cirugía Oral y Maxilofacial. Hospital San Pedro, Logroño.

3 Facultativo Especialista de Área en el Departamento de Cirugía Oral y Maxilofacial. Hospital Universitario Miguel Servet, Zaragoza.
} 


\section{INTRODUCCIÓN}

Las malformaciones vasculares son dilataciones anormales de los vasos con un endotelio no proliferativo, que aparecen y se desarrollan en la edad adulta, pero que suelen ser congénitas. Se clasifican en malformaciones de alto flujo (malformaciones arteriovenosas) y bajo flujo (capilares, venosas y linfáticas) ${ }^{1}$. Las malformaciones de alto flujo son debidas a una comunicación entre una arteria y una vena, lo que produce un aumento de presión con dilatación de la vena (arterialización) y de la arteria suplente, produciéndose una degeneración de la pared de ambos vasos. En la región cérvico-facial las fístulas arteriovenosas tienen un curso poco predecible y no tienen un tratamiento claramente establecido.

\section{MATERIAL Y MÉTODO}

El caso descrito corresponde a una mujer de 41 años, sin otros antecedentes de interés, conocida y en seguimiento por nuestro servicio desde 2010 , por presentar crecimiento de una malformación A-V congénita en región submandibular, suelo de boca derecho y lengua. En febrero de 2015, acude a urgencias por presentar elevación del suelo de la boca e importante macroglosia, que le producía disfagia y comienzo de dificultad respiratoria. Se realizó un TAC en el que se observa una malformación A-V de alto flujo que ocupa el suelo de la boca y toda la lengua de $6 \times 4,5 \times 4,4 \mathrm{~cm}$, produciendo una importante disminución de la columna aérea oro e hipofaríngea. Depende de las arterias facial y lingual derechas principalmente, con grandes dilataciones venosas que drenan en la vena yugular interna izquierda. Tras tratamiento médico con corticoides intravenosos, presentó disminución del tamaño y mejoría clínica. Dado el riesgo vital de la cirugía, además de las importantes secuelas posquirúrgicas, la paciente desestimó la cirugía y se decidió seguir con tratamiento expectante (Figura 1).

\section{RESULTADOS}

Tras explicar las posibilidades de tratamiento en 2010 y tras episodio en febrero de 2015, se decidió conjuntamente con la paciente, seguir tratamiento expectante. En septiembre de 2015, acude de nuevo por dolor y episodio de aumento de tamaño de 72 horas de evolución, sin disnea, ni disfagia en ese momento. Se realiza TAC y arteriografía en las que se observa una masa de mayor tamaño de $82 \times 57 \times 75$ $\mathrm{mm}$, irrigada por ramas de las arterias carótidas externas izquierda y derecha, con trombosis aguda del componente venoso de la malformación. Se comenta el caso con radiología intervencionista, y se plantea de nuevo a la paciente la cirugía radical como único tratamiento curativo. Dado la trombosis espontánea de la malformación A-V y la mejoría clínica de la paciente con discreta disminución de tamaño, se decide continuar con tratamiento conservador expectante. Actualmente, la paciente sigue con importante macroglosia y elevación del suelo de la boca que le produce algo de disnea en

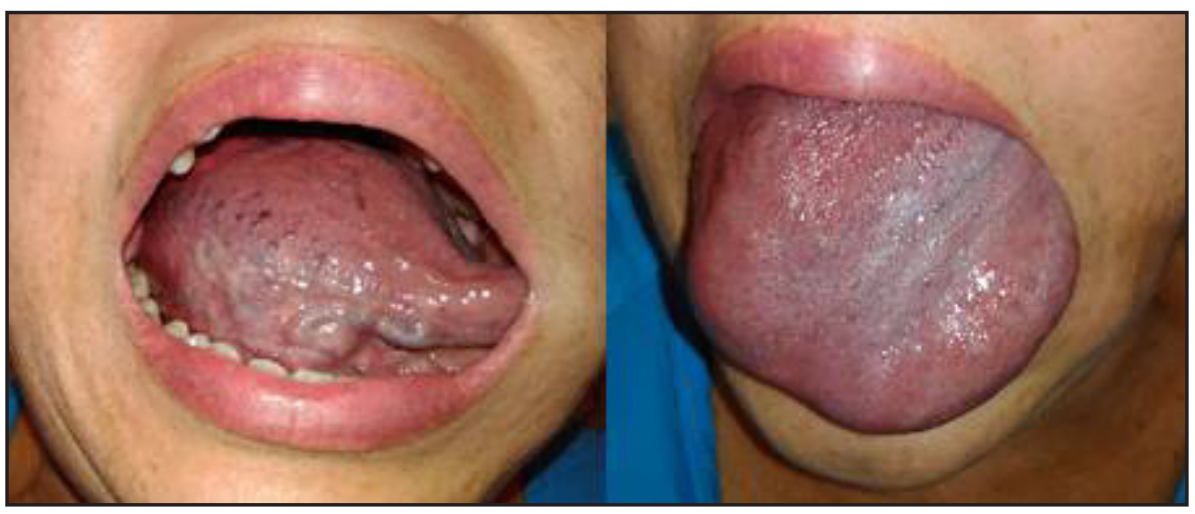

Figura 1. Imagen de la malformación arteriovensa. 


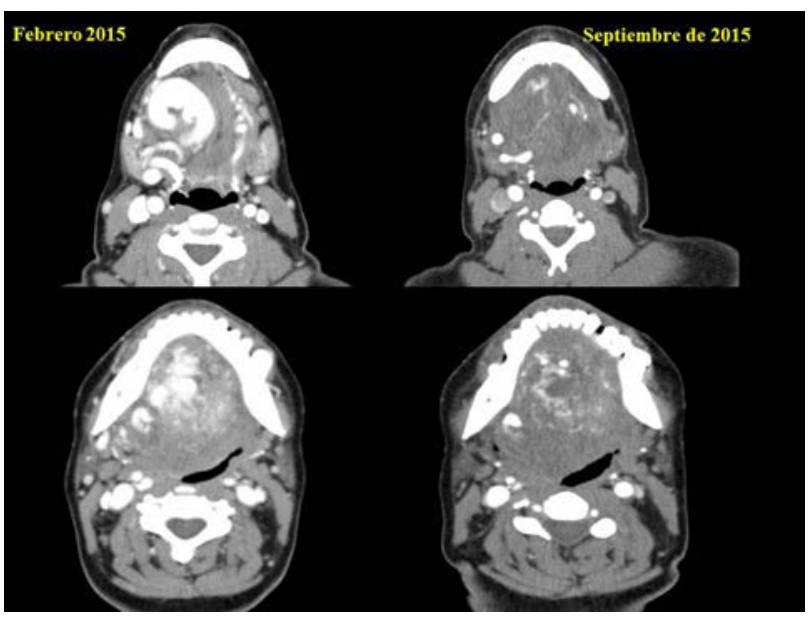

Figura 2. Imágenes posteriores a trombosis espontánea de la lesión.

decúbito y le dificulta la deglución. Pero, desde septiembre de 2015, tras producirse trombosis espontánea del componente venoso, la paciente está clínicamente estable y puede realizar una vida normal, sin presentar ningún nuevo episodio de aumento de tamaño de la lesión (Figura 2).

\section{DISCUSIÓN}

El tratamiento de las malformaciones $A-V$ de alto flujo sigue siendo controvertido, la cirugía tiene una tasa de complicaciones muy alta y la recurrencia es muy frecuente. Existen casos en los que se ha realizado tratamiento quirúrgico en dos pasos, ligando la carótida externa en una primera cirugía, para así reducir el flujo sanguíneo, y poder, en un segundo tiempo, quitar la masa completamente disminuyendo el sangrado ${ }^{1}$. Ligar sólo la carótida externa no es efectivo ya que facilita la nutrición a través de vasos que son inaccesibles quirúrgicamente, y la necrosis de una parte de la tumoración hace más fácil el flujo de sangre dentro de los tejidos de alrededor a través de otros canales². En otros casos, se apuesta por realizar embolización de la carótida externa previa a la cirugía, para así disminuir el flujo sanguíneo antes de la resección quirúrgica. La embolización sola, de todos los vasos nutrientes de la fístula no es efectiva, ya que parte de la lesión es hemodinámicamente inactiva e inaccesible a los émbolos, quedando áreas residuales, que debido a la hipoxia y al aumento de factores de crecimiento, llevan a la recurrencia ${ }^{3,4}$. Se han descrito varias técnicas de embolización, con etanol, coils, n-BCA, Onyx, pero no hay consenso sobre cuál es la mejor, ya que todas tienen sus riesgos y complicaciones ${ }^{5,6}$. Se sabe que el control de las lesiones es mejor cuando el material consigue penetrar y obliterar el nido de vasos (la zona central), por eso nuevos materiales se están investigando, aunque tienen el riesgo de viajar a zonas distantes y producir isquemia ${ }^{4,7}$. El etanol es un material esclerosante que ha demostrado, en algunos casos, ablación permanente de lesiones arteriovenosas sin necesidad de tratamientos posteriores, pero éste no funciona en todas las malformaciones $A-V$, sobre todo en las que tienen una vena dominante con flujo de salida ${ }^{8}$, como en nuestro caso; además, las complicaciones que pueden surgir de su uso son graves, como son la necrosis y el colapso cardiopulmonar ${ }^{9}$. La cirugía de resección 48-72 horas después de realizar la embolización parece ser la mejor opción, pero se han descrito tasas de recurrencia de hasta el $80 \%$ a pesar de realizar cirugía tras embolización. También se ha descrito el tratamiento con radioterapia como medida extrema, para disminuir el tamaño en caso de no poder hacer nada más y existir riesgo vital para el paciente ${ }^{10}$. En teoría, la radiación afecta al epitelio sinusoidal, de manera que los vasos tienden a colapsarse. Sin embargo las malformaciones A-V de alto flujo tienen células maduras que son resistentes a la radioterapia, limitando su acción, produciendo necrosis y riesgo de malignización². 


\section{CONCLUSIÓN}

En la región cérvico facial las malformaciones $A-V$ son raras y siguen siendo un tema controvertido. La principal etiología a este nivel son los traumatismos, seguido de infecciones, pubertad, embarazo, pero también es frecuente que sean espontáneas de origen congénito ${ }^{2}$. El tratamiento es controvertido, y no está claramente establecido, es una decisión tomada entre paciente, cirujano y radiólogo intervencionista. El único tratamiento curativo al día de hoy, es una escisión completa radical de toda la masa, con los grandes riesgos que ésta compete. El primero, la alta tasa de sangrado intraoperatorio (a veces incontrolable por la presencia de estructuras vitales como las arterias carótida, vertebral y subclavia, y las venas yugulares). Otro riesgo a asumir, es la probable recidiva, debido a que depende de gran cantidad de vasos y es necesaria una resección radical de la

\section{BIBLIOGRAFÍA}

1. Dieng Pa, Ba PS, Gaye M, Diatta S, Diop MS, Sene E, Ciss AG, Ndiaye A, Mdiaye M. Giant arteriovenous malformation of the neck. Case Rep Vasc Med 2015; 2015: 124010.

2. Bailey BM, Thomas AA. An arterui-venous fistula of the floor of mouth and tongue. $\mathrm{Br} \mathrm{J} \mathrm{Oral}$ Maxillofac Surg 1984; 22(6): 431-8.

3. González-García R, Rubio-Correa I, Moreno-García C. Massive glosso-cervical arteriovenous malformation: The rationale for a challenging surgical resection. $J$ Clin Exp Dent 2014; 6(4): e456-9.

4. Fowell $\mathrm{C}$, Jones R, Nishikawa $\mathrm{H}$, Monaghan $A$. Arteriovenous malformations of the head and neck: current concepts in management. Br J Oral Maxillofac Surg 2016; 54(5): 482-7.

5. McMillan K, Dunphy L, Nishikawa H, Monaghan A. Experiences in managing arteriovenous malformations of the head and neck. $\mathrm{Br} J$ Oral Maxillofac Surg 2016; 54(6): 643-7.

6. Pekkola J, Lappalainen K, Vuola P, Klockars T, Salminen P, Pitkaranta A. Localization and malformación. Y por otro lado, las secuelas estéticas derivadas, debido a la gran desfiguración que produce la exéresis, necesitando además reconstrucción con colgajos microvascularizados o pediculados, ya que los colgajos libres producen cambios en la vascularización y fibrosis que contribuyen a un ambiente isquémico con la consiguiente recidiva ${ }^{3}$.

Como en nuestro caso, es posible optar por un tratamiento expectante, evitando maniobras agresivas. En nuestra paciente, desde la trombosis venosa espontánea hace 12 meses, a pesar que sigue con importante macroglosia y disnea en decúbito, no ha habido aumento de tamaño, ni sangrado de la lesión. Aportamos este caso, como posible evolución de las malformaciones $A-V$ si se decide un tratamiento expectante en lugar de realizar una cirugía radical. Más casos y mayor tiempo de seguimiento son necesarios, para valorar la evolución a largo plazo de estas lesiones.

treatment of lingual venous and arteriovenous malformations. AJNR Am J Neuroradiol 2013; 34(1): 198-204.

7. Karim AB, Lindsey $S$, Bovino $B$, Berenstein A. Oral surgical procedures performed safely in patients with head and neck arteriovenous malformations: A retrospective case series of 12 patients. J Oral Maxillofac Surg 2016; 74(2): 255.

8. Su L, Wang D, Han Y, Wang Z, Zheng L, Fan X. Absolute ethanol embolization of infiltratingdiffuse extracranial arteriovenous malformations in the head and neck. Eur J Vasc Endovasc Surg 2015; 50(1): 114-21.

9. Karim AB, Lindsey S, Bovino B, Berenstein A. Oral surgical procedures performed safely in patients with head and neck arteriovenous malformations: A retrospective case series of 12 patients. J Oral Maxillofac Surg 2016; 74(2): 255.

10. Rudman RA, Clark WJ. A large vascular malformation of the tongue treated with radiation therapy. J Oral Maxillofac Surg 1997; 55(5): 509-14.

Dirección : Marina Alexandra Gavín Clavero C/Doctor Lozano Monzón 50006. Zaragoza (España). E mail: marinagvncla@gmail.com 